\title{
Quantum critical phenomena in the Schrödinger formulation: mapping to classical lattices
}

\author{
Ricardo A. Sauerwein ${ }^{1}$, Sabre Kais * \\ Department of Chemistry, Purdue University, West Lafayette, IN 47907, USA
}

Received 9 October 2000; in final form 28 November 2000

\begin{abstract}
We present a path integral formalism to obtain the statistical mechanics transfer matrix of the system which leads to a straightforward mapping between the quantum problem and an effective classical lattice system. Thus, the quantum criticality of atomic and molecular systems can be studied by the standard statistical mechanics methods. We illustrate this general technique by presenting detailed calculations for the screened Coulomb potential. (c) 2001 Elsevier Science B.V. All rights reserved.
\end{abstract}

\section{Introduction}

Phase transitions and critical phenomena continue to be a subject of great interest in many fields [1]. A wide variety of physical systems exhibit phase transitions and critical phenomena, such as liquid-gas, ferromagnetic-paramagnetic, fluid-superfluid, conductor-superconductor and the list continues to expand [2]. Over the last few decades, a large body of research has been done on this subject, mainly using classical statistical mechanics. However, quantum phase transitions have attracted much interest in recent years. These transitions are zero temperature transitions tuned by parameters in the Hamiltonian [3,4]. Examples from condensed matter physics include the magnetic transitions of cuprates, superconductor-

\footnotetext{
${ }^{*}$ Corresponding author.

E-mail address: kais@power1.chem.purdue.edu (S. Kais).

${ }^{1}$ On leave from Universidade Federal de Santa Maria, Santa Maria, RS, Brazil.
}

insulator transitions in alloys, metal-insulator transitions and the Quantum-Hall transitions $[4,5]$.

In the field of atomic and molecular physics, the analogy between symmetry breaking of electronic structure configurations and quantum phase transitions has been established at the large dimensional limit [6]. The mapping between symmetry breaking and mean-field theory of phase transitions was shown by allowing the nuclear charge $Z$, the parameter which tunes the phase transition, to play a role analogous to temperature in classical statistical mechanics [7]. The study of quantum phase transitions and critical phenomena continues to be of increasing interest in the field of atomic and molecular physics. This is motivated by the recent theoretical and experimental searches for the smallest stable multiply charged anions $[8,9]$, experimental and theoretical work on the stability of atoms and molecules in external electric and magnetic fields [10,11], design and control electronic properties of materials using artificial atoms [12], the study of selectively breaking 
chemical bonds in polyatomic molecules [10] and phase transitions of finite clusters [13].

To treat quantum phase transitions and critical phenomena, it seems that Feynman's path integral is the natural choice. In this approach, one can show that the quantum partition function of the system in $d$ dimensions looks like a classical partition function of a system in $d+1$ dimensions where the extra dimension is the time [3]. Upon doing so, and allowing the space and time variables to have discrete values, we turn the quantum problem into an effective classical lattice problem. Quantum phase transitions occur at zero temperature, $T=0$. At this limit the free energy becomes the ground state energy and the various thermal averages become the ground state expectation values. With the partition function, we can use standard statistical mechanics methods, such as the finite size scaling, to get all the quantities of interest for investigating quantum phase transitions. In the following sections, we will show how to carry out the mapping between the quantum problem and an effective classical space-time lattice, give an example to illustrate how calculate the different critical parameters, and then give discussion and conclusions.

\section{Mapping quantum problems to lattice systems}

In the path integral approach, the probability amplitude $K\left(q^{\prime}, t^{\prime} ; q, t\right)$ of a particle initially localized at position $q$ and time $t$ to be found at position $q^{\prime}$ at time $t^{\prime}$ is given by

$K\left(q^{\prime}, t^{\prime} ; q, t\right)=\int_{q(t)=q}^{q\left(t^{\prime}\right)=q^{\prime}} \mathscr{D} q \mathrm{e}^{(i / h) S[q(t)]}$,

where the symbol $\int_{q(t)=q}^{q\left(t^{\prime}\right)=q^{\prime}} \mathscr{D} q$ stands for integration over all trajectories connecting the space-time point $(q, t)$ to $\left(q^{\prime}, t^{\prime}\right)$, and $S[q(t)]$ is the classical action for a given trajectory $q=q(t)[14,15]$.

The analytical continuation of the probability amplitude to imaginary time $t=-\mathrm{i} \tau$ of closed trajectories, $q(t)=q\left(t^{\prime}\right)$, is formally equivalent to the quantum partition function $Z(\beta)$, with the inverse temperature $\beta=-\mathrm{i}\left(t^{\prime}-t\right) / \hbar$ [16]. In practical calculations it is common to use the path integral lattice definition. In this discrete time approach, the quantum partition function reads

$$
\begin{aligned}
Z(\beta)= & \lim _{\Delta \tau \rightarrow 0}\left(\frac{m}{2 \pi \Delta \tau \hbar}\right)^{N_{\tau} / 2} \int \prod_{\ell=0}^{N_{\tau}} \mathrm{d} q_{\ell} \\
& \times \exp \left[-\frac{\Delta \tau}{\hbar}\left(\sum_{\ell=0}^{N_{\tau}-1} m \frac{\left(q_{\ell+1}-q_{\ell}\right)^{2}}{2(\Delta \tau)^{2}}\right.\right. \\
& \left.\left.+\lambda \sum_{\ell=0}^{N_{\tau}-1} \frac{V\left(q_{\ell}+1\right)+V\left(q_{\ell}\right)}{2}\right)\right]
\end{aligned}
$$

where $\lambda$ is the strength of the potential $V(q), \Delta \tau=\beta / N_{\tau}$ is the regular grid spacing between $N_{\tau}$ points along the imaginary time axis indexed by $\ell=0,1, \ldots, N_{\tau}$. The closed path is made by a periodic boundary condition in the time direction such that $q_{N_{\tau}}=q_{0}$. Since we are considering a quantum phase transition for a system at its ground state energy, we must consider only the case of $\beta \rightarrow \infty$.

Now we introduce the discretization also in the space direction. Thus, the totally discrete model can be used as a new scheme to study quantum particles in the presence of a potential with a point singularity. This kind of potential cannot be described by the path integral formalism with a finite $\Delta \tau$ within the so-called primitive approximation [17]. Besides the discretization, the present scheme depends upon the thermodynamic and continuum limits which we will discuss later.

This way the position in time slice $\ell$ is given by

$q_{i_{\ell}}=q_{0}+i_{\ell} \Delta L \quad$ with $i_{\ell}=1,2, \ldots, N_{q}$,

where $\Delta L$ is the regular grid spacing of the position axis which has a total of $N_{q}$ points and $q_{0}$ is a constant used to adjust the origin of the coordinate system. Moreover, the size of the space is limited by $L=N_{q} \Delta L$.

Now we concentrate on the properties of the two-dimensional lattice, the space-time lattice. The partition function, Eq. (2), shows that there is coupling only in the time direction and only between nearest neighbor time slices. This allows us to use the statistical mechanics technique of writing the partition function $Z$ of the finite system as the trace of a matrix $T$ to the power $N_{\tau}$

$Z(\Delta L, \Delta \tau)_{N_{q}, N_{\tau}}=\operatorname{Tr}\left(T^{N_{\tau}}\right)$. 
The matrix $T$ is called the transfer matrix. Its elements are given by

$$
\begin{aligned}
T\left(i_{\ell}, i_{\ell+1}\right)= & \Delta L\left(\frac{m}{2 \pi \hbar \Delta \tau}\right)^{1 / 2} \\
& \times \exp \left\{-\frac{\Delta \tau}{\hbar}\left[\frac{m}{2}\left(\frac{\Delta L}{\Delta \tau}\right)^{2}\left(i_{\ell+1}-i_{\ell}\right)^{2}\right.\right. \\
& \left.\left.+\lambda \frac{V_{i_{\ell}+1}+V_{i_{\ell}}}{2}\right]\right\}
\end{aligned}
$$

where $V_{i_{\ell}}=V\left(q_{\ell}\right)$ is the potential energy of time slice $\ell$ evaluated at the space point $i_{\ell}$.

The above transfer matrix can be seen as a transfer matrix of a classical pseudo-system. The Hamiltonian of the pseudo-system reads

$\mathscr{H}=\sum_{\ell=1}^{N_{\tau}} \mathscr{H}_{\ell, \ell+1}$

where

$\mathscr{H}_{\ell, \ell+1}=\frac{m}{2}\left(\frac{\Delta L}{\Delta \tau}\right)^{2}\left(i_{\ell+1}-i_{\ell}\right)^{2}+\lambda \frac{V_{i_{\ell+1}}+V_{i_{\ell}}}{2}+C$

with $C=-\hbar \ln \left\{[m /(2 \pi \hbar \Delta \tau)]^{1 / 2} \Delta L\right\} / \Delta \tau$ being a constant independent of the state of the lattice. Then, the partition function of the classical pseudo-system becomes

$T\left(i_{\ell}, i_{\ell+1}\right)=\exp \left[-(\Delta \tau / \hbar) \mathscr{H}_{\ell, \ell+1}\right]$.

If the classical pseudo-system Hamiltonian were independent of $\Delta \tau$, the classical pseudo-system would behave as a statistical mechanics lattice system with inverse temperature $\Delta \tau / \hbar$.

In order to complete the mapping between the quantum problem and the classical pseudo-system, one must address the problems of both the continuum and the infinite limits. The ground state properties of the original system are obtained by taking both the continuum limit, $(\Delta \tau, \Delta L \rightarrow 0)$, and the thermodynamic limit, $(\beta, L \rightarrow \infty)$. We assume that in principle this limit can be performed in two steps. First, we take the thermodynamic limit $N_{\tau}, N_{q} \rightarrow \infty$, and second, we take the continuum limit $\Delta \tau, \Delta L \rightarrow 0$. From the definition of the finite transfer matrix, (5), we see that both $\Delta \tau$ and $\Delta L$ always appear inside the factor $\left(m \Delta L^{2} / \Delta \tau \hbar\right)$. So it is clear that we cannot choose $\Delta L$ and $\Delta \tau$ independently, otherwise we could end up with an undefined transfer matrix when the continuum limit is taken. This problem can be overcome by taking

$$
\frac{m \Delta L^{2}}{\Delta \tau \hbar}=1 \Rightarrow \Delta L\left(m \frac{\Delta L}{\Delta \tau}\right)=\hbar .
$$

The above expression can be seen as a quantization of the action in the semi-classical theory. Also, this same dependency $\Delta \tau=\Delta L^{2}$ is imposed in order to get the diffusion equation when taking the continuum limit of the Brownian motion of particles in a lattice [22].

The number of points along the imaginary time $N_{\tau}$ does not appear explicitly in any relevant quantity. The requirement $N_{\tau} \rightarrow \infty$ is necessary in order to have a finite partition function, Eq. (4), dominated by the leading eigenvalue of the transfer matrix $a_{0}\left(\Delta L, \Delta \tau ; N_{q}\right)$. We can see how $N_{\tau}$ is canceled when evaluating the ground state energy of the system $E_{0}$. In the lattice system, $E_{0}$ is the free energy per time slice which is related to the partition function and consequently to $a_{0}(\Delta L, \Delta \tau$; $\left.N_{q}\right) \quad$ by $\quad \exp \left(-N_{\tau} \Delta \tau E_{0}\right)=Z(\Delta L, \Delta \tau)_{N_{q}, N_{\tau}} \approx a_{0} \quad(\Delta L$, $\left.\Delta \tau ; N_{q}\right)^{N_{\tau}}$. Since $N_{\tau}$ appears in both ends of this equation the energy is given by $E_{0}=-\ln \left(a_{0}\right.$ $\left.\left(\Delta L, \Delta \tau ; N_{q}\right)\right) / \Delta \tau$. Thus the discretization number which actually sets the size of the finite system is $N_{q}$. Setting $N_{q}=N$ and considering implicitly that $N_{\tau}$ goes also to infinity when taking the thermodynamic limit, the original partition function for $\beta \rightarrow \infty$ is recovered by taking the limit

$$
Z(\beta)=\lim _{\substack{\Delta L \rightarrow \varepsilon \\ \Delta \tau=\Delta L^{2}}}\left[\lim _{N \rightarrow \infty} Z(\Delta L, \Delta \tau)_{N}\right],
$$

where $\epsilon$ is an arbitrarily small number.

The inner limit of the above expression $N \rightarrow \infty$ represents the 'thermodynamic limit' for our system. In previous studies [7] of quantum phase transitions, it was proposed that the role of the 'thermodynamic limit' be played by the number of terms in the truncated basis set used to expand the exact wave function. The thermodynamic limit in the present study seems to be more natural, since it is given by an extensive parameter of the 
pseudo-system; however, we believe that the two definitions are essentially the same. Coalson [18] showed the connection between Fourier coefficients and the discrete path integration. The number of points $N$ of the discrete path integral is related to the number of plane waves, i.e., the number of terms of a truncated basis set used to represent the states of the quantum system.

The phase transition is driven by the continuous parameter $\lambda$. There are two possible phases. These corresponding to the presence or absence of a stable bound ground state energy of the quantum system. In the present formalism, these two phases are identified by localized or delocalized particles. As in ordinary statistical mechanics lattice systems, the transition point can be identified as the divergence or discontinuity point in the correlation length. The two point correlation function can be defined as

$C_{\lambda}(\ell)=\left\langle i_{0} i_{\ell}\right\rangle-\left\langle i_{0}\right\rangle^{2}$,

where the symbol $\langle\cdots\rangle$ stands for the statistical mechanics ensemble or 'thermal' average. Its asymptotic behavior for large systems is given by

$C_{\lambda}(\ell) \approx \mathrm{e}^{-\ell \Delta \tau / \xi(\lambda)}$,

where $\xi(\lambda)$ is the correlation length. The physical meaning of this quantity is the distance along the imaginary time within which the time slices are correlated. It can be shown [2] that in large systems the correlation length can be evaluated by

$\xi(\lambda)-\frac{1}{\ln \left(a_{1} / a_{0}\right)} \Delta \tau$,

where $a_{0}$ and $a_{1}\left(a_{0}>a_{1}\right)$ are the two leading eigenvalues of the transfer matrix.

Any operator that is diagonal in the discrete point basis set, like the potential energy or any other function of the particle position say $f(q)$, has the ensemble average

$\left\langle f\left(i_{\ell}\right)\right\rangle=\left\langle\psi^{(0)}|f(q)| \psi^{(0)}\right\rangle=\sum_{i=1}^{N} f\left(q_{i}\right)\left|\psi_{i}^{(0)}\right|^{2}$,

where $\psi^{(0)}$ is the transfer matrix leading eigenvector. At the limit $\beta \rightarrow \infty$, this eigenvector is the ground state eigenstate of the quantum system in the discrete representation. So the ensemble average of the lattice system in the thermodynamic limit becomes the ground state quantum average $\left\langle\psi^{(0)}|f(q)| \psi^{(0)}\right\rangle$.

\section{Numerical calculations}

To illustrate this general approach, we will carry out the calculations for the critical parameters of the screened Coulomb potential, $V(r)=$ $-\mathrm{e}^{-r} / r$. This potential is frequently used in quantum calculations and the exact values of the critical parameters $\lambda_{\mathrm{c}}$ for different states are known from finite size scaling calculations [19]. In atomic units, the Hamiltonian can be written as

$\mathscr{H}(\lambda)=-\frac{1}{2} \nabla-\lambda \frac{\mathrm{e}^{-r}}{r}$.

A critical point, $\lambda_{\mathrm{c}}$, is defined as the value of $\lambda$ for which the bound state energy becomes absorbed or degenerate with a continuum [7]. This system is known to exhibit a continuous phase transition for states with zero angular momentum, $l=0$, and first-order phase transition for states with nonzero angular momentum [19].

Hamiltonians with spherical symmetry can be investigated with a radial path integral [20]. Using the above proposed scheme, the calculation is performed by taking $N \rightarrow \infty$ with $\Delta L$ (and $\Delta \tau$ ) fixed. Excluding the origin with the radial grid $r_{i}=i \Delta L$, with $i=1,2, \ldots, N$, the potential is effectively bounded from below. Numerically we found that no matter how small we take $\Delta L$, for a sufficiently large value of $N$, we get the same limiting value.

The transfer matrix for the radial path integral equation is

$T_{\mathrm{rad}}\left(i_{\ell}, i_{\ell+1}\right)=M_{l+(3 / 2)}\left(i_{\ell}, i_{\ell+1}\right) T\left(i_{\ell}, i_{\ell+1}\right)$,

where $T\left(i_{\ell}, i_{\ell+1}\right)$ is the same transfer matrix defined in Eq. (5) but with the potential terms given by the radial discretization rule $V_{i_{\ell}}=-\lambda \exp \left[i_{\ell} \Delta L\right]=$ $\left(i_{\ell} \Delta L\right)$. The factor $M_{l+(3 / 2)}\left(i_{\ell}, i_{\ell+1}\right)$ is introduced by the discrete radial functional weight

$$
\begin{aligned}
M_{l+(3 / 2)}\left(i_{\ell}, i_{\ell+1}\right)= & \sqrt{2 \pi \frac{\Delta L^{2}}{\Delta \tau} i_{\ell} i_{\ell+1}} \exp \left[-\frac{\Delta L^{2}}{\Delta \tau} i_{\ell} i_{\ell+1}\right] \\
& \times I_{l+(3 / 2)}\left(\frac{\Delta L^{2}}{\Delta \tau} i_{\ell} i_{\ell+1}\right),
\end{aligned}
$$


where $I_{l+(3 / 2)}$ is the modified Bessel function. In the context of the pseudo-system, the pre-factor $M_{\ell+(3 / 2)}\left(i_{\ell}, i_{\ell+1}\right)$ plays the role of a centrifugal potential which leads to another coupling between consecutive time slices.

In order to study the properties of the system we fix $\Delta L$ and take $\Delta \tau=\Delta L^{2}$. Then we numerically calculate the two leading eigenvalues with their respective eigenvectors for different values of $\lambda$ and different matrix sizes $N$. The correlation length $\xi^{(N)}(\lambda)$ is then readily evaluated using Eq. (15). An 'order parameter' can be defined as the average radial coordinate scaled by the system size $R / L=\left\langle i_{\ell}\right\rangle / N$. Klaus and Simon [23] showed that short range potentials in three dimensions, such as the Yukawa potential, do not have normalizable ground state eigenfunctions at transition points. Thus, for the case $l=0$, we can foresee that $R / L=R^{*} / L \neq 0$ at the transition point $\left(R^{*}\right.$ is $R$ at the transition point); although in the thermodynamic limit, this magnitude identifies the two different symmetries. A localized state has $R / L$ $<R^{*} / L$, and a nonlocalized state has $R / L>R^{*} / L$. In the case $l=1$, the centrifugal term makes the Yukawa potential effectively long range. Thus the ground state wave function of the localized states are normalizable even at the transition point. Hence, the distinction between the two symmetries becomes clearer. In the thermodynamic limit, $R / L=0$ identifies localized states while $R / L \neq 0$ identifies nonlocalized states.

The effect of the grid spacing and the system size, in the localization of the pseudo-critical point, is summarized in Fig. 1. Each curve corresponds to a different value of $\Delta L\left(\Delta \tau=\Delta L^{2}\right)$. Notice that there is indeed a 'thermodynamic limit' since as $N \rightarrow \infty$ the pseudo-critical points $\lambda_{\mathrm{c}}^{(N)}$ converges towards a definite value. This value can depend on the grid spacing. In principle, we could think that the smaller the grid spacing the closer its thermodynamic extrapolation will be to the actual transition point $\lambda_{\mathrm{c}}$. However, our results show that the extrapolated value of $\lambda_{\mathrm{c}}^{(N)}$ for $0.01 \leqslant \Delta L \leqslant 0.06$ leads to the same accurate value $\lambda_{\mathrm{c}} \approx 0.840(1)$ which is in perfect agreement with previous calculation using the truncated basis set scheme [19].

In Fig. 2 we show the correlation length $\xi$ and the order parameter $R / L$ as a function of $\lambda$ for

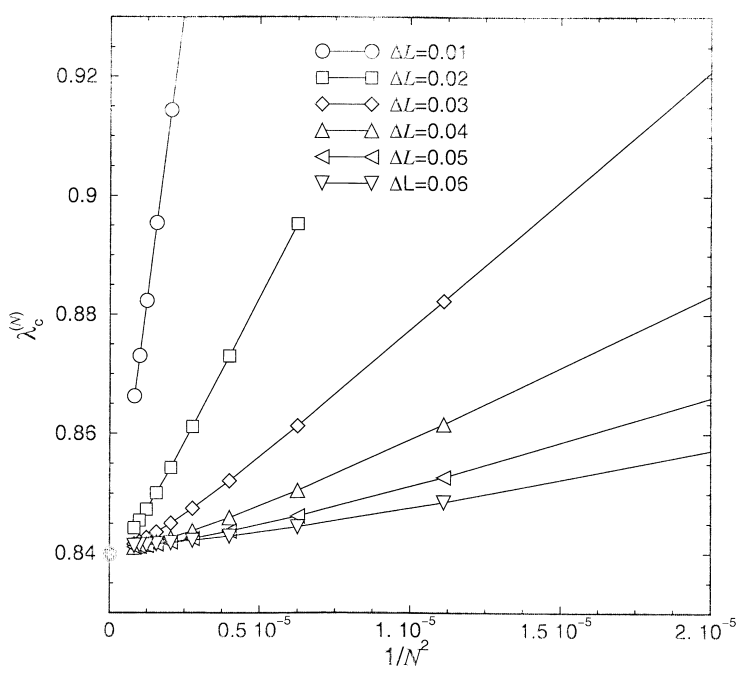

Fig. 1. The pseudo-critical points, $\lambda_{\mathrm{c}}^{(N)}$ vs $1 / N^{2}$ for the ground state of the Yukawa potential for different grid spacing $\Delta L$. The exact value of $\lambda_{\mathrm{c}}$ is also shown by a dot.

fixed values of $\Delta L=0.05$ and $\Delta \tau=\Delta L^{2}$. Each curve in these figures corresponds to calculations with different values of $N$. For $l=0$, there is a clear peak in the correlation length $\xi^{(N)}(\lambda)$ which scales with $L^{2}$. The position of the peak for each lattice size determines the pseudo-critical transition point $\lambda_{\mathrm{c}}^{(N)}$. The corresponding order parameter $R / L$ curves show that this transition is continuous or, of the second-order type. For angular momentum $l=1$, the correlation length $\xi$ and the the order parameter $R / L$ show a different transition: a first-order phase transition. There is a clear peak in the correlation length $\xi^{(N)}(\lambda)$ curves which scales with $L^{2}$. Comparing the case $l=0$ and $l=1$ we see that the behavior of the correlation length near the transition point is qualitatively different. This difference also appears in the order parameter. We see in Fig. 2 that the $R / L$ curves tend to a step-like function as the system size grows.

In order to complete the comparison between the two different types of transitions, we plot in Fig. 3 the energy $E$, the first derivative $\mathrm{d} E / \mathrm{d} \lambda$, the correlation length $\xi$, and the order parameter $R / L$ for $l=0$ and $l=1$ with the system size $N=2000$ and $\Delta L=0.05$. All of these quantities are plotted as a function of the scaled parameter 

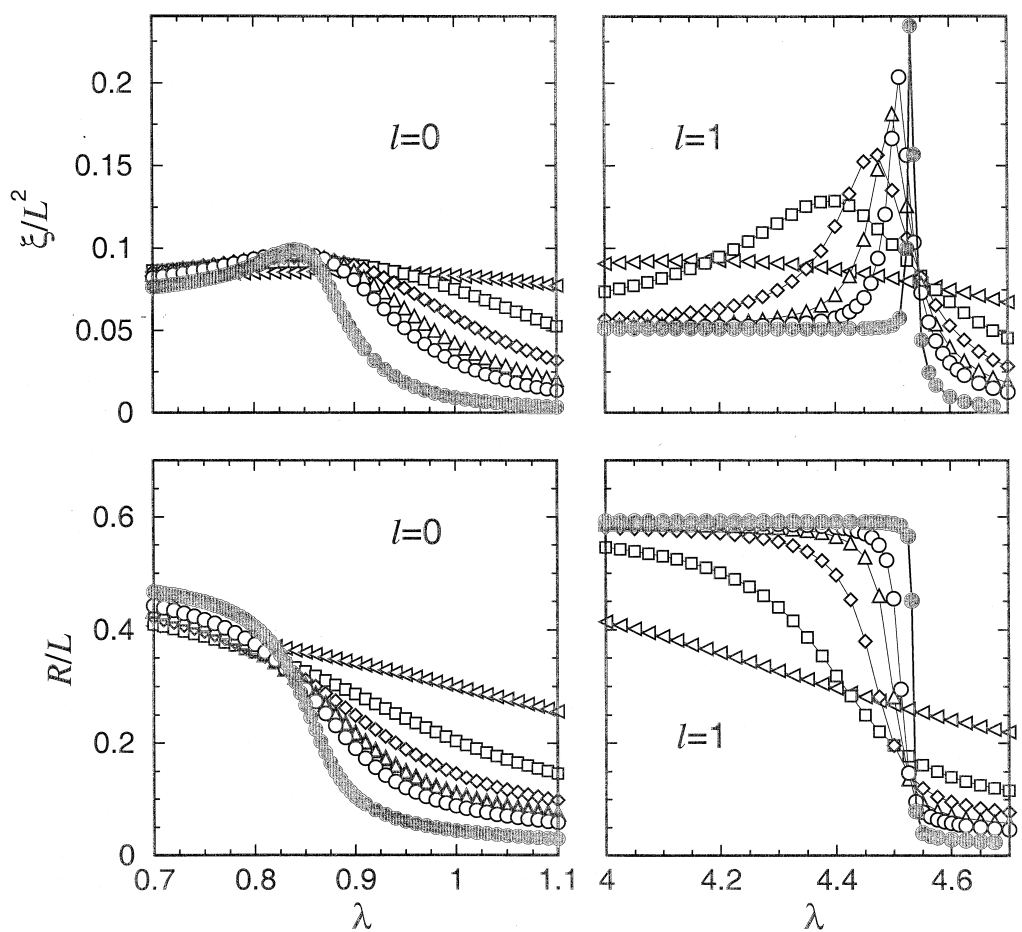

Fig. 2. The scaled correlation length $\xi / L^{2}$ and the order parameter $R / L$ vs $\lambda$ for the Yukawa potential with $l=0$ and $l=1$ for increasing grid points $N=200(\triangleleft), 400(\square), 600(\diamond), 800(\triangle), 1000(\bigcirc)$ and $2000(\bullet)$, using $\Delta L=0.05$.

$\left(\lambda-\lambda_{\mathrm{c}}^{(N)}\right) / \lambda_{\mathrm{c}}^{(N)}$ where $\lambda_{\mathrm{c}}^{(N)}$ is the pseudo-critical value for each finite system. The nature of the phase transitions is now very clear: continuous for $l=0$ and first-order for $l=1$.

The study of phase transitions and critical phenomena show that physical systems as different as fluids and anisotropic ferromagnets can be grouped into the same universality class. Remarkably few universality classes are defined by the values of a set of critical exponents which are given by the asymptotic behavior of some quantities near the critical point [2]. As we have mapped our original problem into a lattice system we are tempted to ask if this pseudo-system has such a universal behavior. The critical exponents associated with the correlation length $v$ and the specific heat $\alpha$ are, respectively, defined as

$\xi \approx\left|\lambda-\lambda_{\mathrm{c}}\right|^{-v}$ and $E_{0} \approx\left|\lambda-\lambda_{\mathrm{c}}\right|^{2-\alpha}$.

Notice that in our system, $\lambda$ plays the role of the temperature. Thus, the second derivative of the ground state energy of the quantum system (or the free energy of the lattice model) with respect to $\lambda$ is analogous to the specific heat in thermodynamic models.

Although the calculations in the present work are performed in a finite system, we can use the scaling invariance property of systems near criticality to extrapolate our data to the thermodynamic limit. This extrapolation technique is based on the theory of finite size scaling [21]. We used the algorithm of Bulirsh and Stoer to obtain the extrapolated values of all the magnitudes [7]. The results for the critical parameters are given in Table 1.

\section{Discussion and conclusions}

We have shown with the use of Feynman's path integral approach that by allowing the space and time variables to have discrete values, the quantum 

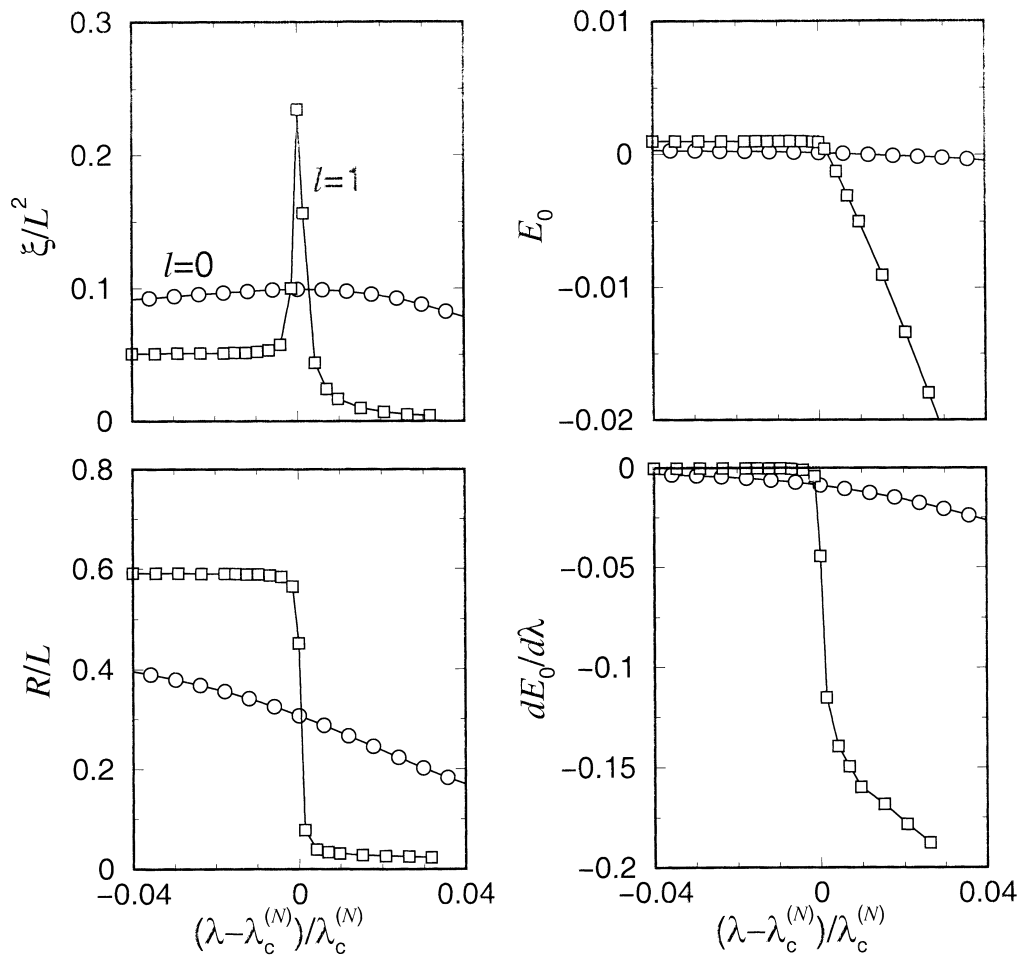

Fig. 3. Comparison of the bound state energy $E$, the first derivative $\mathrm{d} E / \mathrm{d} \lambda$, the scaled correlation length $\xi / \beta N$, and the order parameter $R / L$ as a function of the scaled $\lambda$ for the Yukawa potential at second-order phase transition, $l=0(O)$ and first-order transition, $l=1(\square)$.

Table 1

Critical parameters for the Yukawa potential for $l=0$ and $l=1$

\begin{tabular}{lll}
\hline Quantity & $l=0$ & $l=1$ \\
Transition-order & Second-order & First-order \\
\hline$\lambda_{\mathrm{c}}$ & $0.840(1)$ & $4.540(2)$ \\
$\alpha$ & $0.00(1)$ & $0.99(2)$ \\
$v$ & $1.00(1)$ & $0.500(5)$ \\
\hline
\end{tabular}

problem can be mapped to an effective classical lattice problem. The lattice can also be used to remove an existing point singularity of the original quantum problem without compromising its critical behavior. Thus, the quantum criticality of the original system can be studied by the powerful statistical mechanics methods developed for lattices. In particular, the Monte Carlo techniques can be directly applied. Hence, opening the possibility of studying larger atomic and molecular systems. With this analogy we have obtained very accurate critical parameters for the screened Coulomb potential for both first-order and continuous phase transitions.

The field of quantum critical phenomena in atomic and molecular physics is still in its infancy and there are many open questions about the interpretations of the results including whether or not these quantum phase transitions really do exist. The possibility of exploring these phenomena experimentally in the field of artificial atoms [24] offers an exciting challenge for future research. This approach is general and might provide a powerful way in determining critical parameters for the stability of atomic and molecular systems in external fields, for selectively breaking chemical bonds and for design and control electronic properties of materials using artificial atoms. 


\section{Acknowledgements}

We would like to acknowledge the financial support of the National Science Foundation and the Office of Naval Research.

\section{References}

[1] B. Benderson, More Things in Heaven and Earth, A Celebration of Physics at the Millennium, Springer, APS, 1999 , p. 501.

[2] J.J. Binney, N.J. Dowrick, A.J. Fisher, M.E.J. Newman, The Theory of Critical Phenomena: An Introduction to the Renormalization Group, Oxford University Press, Oxford, 1992.

[3] S.L. Sondhi, S.M. Girvin, J.P. Carini, D. Shahar, Rev. Mod. Phys. 69 (1997) 315.

[4] S. Sachdev, Quantum Phase Transions, Cambridge University Press, New York, 1999.

[5] H.L. Lee, J.P. Carini, D.V. Baxter, W. Henderson, G. Gruner, Science 287 (2000) 633.

[6] P. Serra, S. Kais, Phys. Rev. Lett. 77 (1996) 466.

[7] S. Kais, P. Serra, Int. Rev. Phys. Chem. 19 (2000) 97.

[8] M.K. Scheller, R.N. Compton, L.S. Cederbaum, Science 270 (1995) 1160.

[9] X. Wang, L. Wang, Phys. Rev. Lett. 83 (1999) 3402.
[10] R. Rost, J. Nygard, A. Pasinski, A. Delon, Phys. Rev. Lett. 78 (1997) 3093.

[11] Y.P. Kravchenko, M.A. Lieberman, Phys. Rev. A 56 (1997) R2510.

[12] F. Remacle, R.D. Levine, Proc. Natl. Acad. Sci. USA 97 (2000) 553.

[13] R.S. Berry, Nature (London) 393 (1998) 212.

[14] H. Kleinert, Path Integrals, World Scientific, New Jersey, 1995.

[15] N. Makri, Ann. Rev. Phys. Chem. 50 (1999) 167.

[16] M.L. Bellac, Quantum and Statistical Field Theory, Oxford University Press, New York, 1998.

[17] J.M. Thijssen, Computational Physics, Cambridge University Press, Cambridge, 1999.

[18] R.D. Coalson, J. Chem. Phys. 85 (1986) 926.

[19] P. Serra, J.P. Neirotti, S. Kais, Phys. Rev. A 57 (1998) R1481.

[20] C. Grosche, F. Steiner, Handbook of Feynman Path Integrals, Springer, Berlin, 1998.

[21] V. Privman (Ed.), Finite Size Scaling and Numerical Simulations of Statistical Systems, World Scientific, Singapore, 1990.

[22] B.D. Hugues, Random Walks and Random Environments, vol. 1, Random Walks, Clarendon, 1995.

[23] M. Klaus, B. Simon, Ann. Phys. 130 (1980) 251.

[24] L. Jacak, P. Wawrylak, A. Wojs, Quantum Dots, Springer, New York, 1998. 